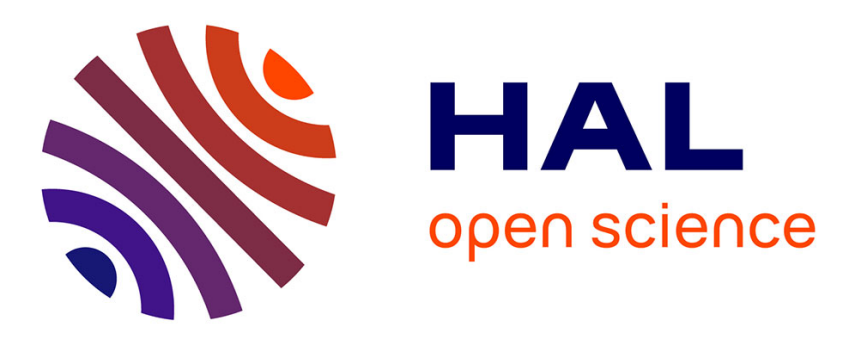

\title{
Signatures métabolomiques par spectrométrie de masse et risque de cancer du sein
}

Lucie Lecuyer, Céline Dalle, Bernard Lyan, Mélanie Pétéra, Marie Lagrée, Adrien Rossary, Aïcha Demidem, Tom Ferreira, Delphine Centeno, Pilar Galan, et al.

\section{To cite this version:}

Lucie Lecuyer, Céline Dalle, Bernard Lyan, Mélanie Pétéra, Marie Lagrée, et al.. Signatures métabolomiques par spectrométrie de masse et risque de cancer du sein. Cancer: Les Actualités de la Recherche en Nutrition, Fondation ARC.; Réseau National Alimentation Cancer Recherche (réseau NACRe). FRA., Nov 2017, Paris, France. 48 p. hal-02735580

\section{HAL Id: hal-02735580 \\ https://hal.inrae.fr/hal-02735580}

Submitted on 2 Jun 2020

HAL is a multi-disciplinary open access archive for the deposit and dissemination of scientific research documents, whether they are published or not. The documents may come from teaching and research institutions in France or abroad, or from public or private research centers.
L'archive ouverte pluridisciplinaire HAL, est destinée au dépôt et à la diffusion de documents scientifiques de niveau recherche, publiés ou non, émanant des établissements d'enseignement et de recherche français ou étrangers, des laboratoires publics ou privés. 


\section{Lucie LÉCUYER}

\section{Signatures métabolomiques par spectrométrie de masse et risque de cancer du sein}

Lucie Lécuyer ${ }^{1}$, Céline Dalle², Bernard Lyan², Mélanie Petera², Marie Lagree², Adrien Rossary², Aicha Demidem², Tom Ferreira ${ }^{2}$, Delphine Centeno ${ }^{2}$, Pilar Galan ${ }^{1}$, Serge Hercberg ${ }^{1,3}$, Mélanie Deschasaux ${ }^{1}$, Valentin Partula ${ }^{1}$, Bernard Srour ${ }^{1}$, Paule Latino-Martel ${ }^{1}$, Emmanuelle Kesse-Guyot ${ }^{1}$, Claudine Manach², Marie-Paule Vasson ${ }^{2}, 4$, Stéphanie Durand ${ }^{2}$, Estelle Pujos-Guillot ${ }^{2}$, Mathilde Touvier ${ }^{1}$

1. Équipe de Recherche en Epidémiologie Nutritionnelle (EREN), CRESS, Inserm U1153, Inra U1125, Cnam, Université Paris 13, Bobigny (NACRe 01)

2. Université Clermont Auvergne, INRA, UMR 1019, Unité de Nutrition Humaine (UNH), CRNH Auvergne, ClermontFerrand (NACRe 33)

3. Département de santé publique, Hôpital Avicenne, Bobigny

4. Centre Anticancer Jean-Perrin, CHU Clermont-Ferrand

l.lecuyer@eren.smbh.univ-paris13.fr

Mots-clés : métabolomique ; cancer du sein ; spectrométrie de masse ; plasma ; étude prospective

I Contexte et objectifs : La métabolomique permet d'étudier l'ensemble des métabolites présents dans un biofluide. Cette étude basée sur une analyse non ciblée en spectrométrie de masse a pour but d'étudier si des profils métabolomiques établis à partir d'un prélèvement sanguin sur des femmes a priori en bonne santé, pourrait contribuer à prédire le risque à long terme de développer le cancer du sein et d'améliorer la compréhension de son étiologie.

I Méthodes : Un cas-témoin niché prospectif a été mené dans la cohorte SU.VI.MAX, incluant 211 cas de cancer du sein et 211 témoins appariés. Les profils métabolomiques ont été établis sur des échantillons de plasma prélevés à l'inclusion, avant l'apparition du cancer, grâce à une analyse non ciblée par spectrométrie de masse (LC-MS). Des modèles de régressions logistiques conditionnelles multivariées ont été calculés sur chaque ion présélectionné grâce à une ANOVA et sur les combinaisons de potentiels biomarqueurs issus d'une Analyse en Composante Principale. Une correction de test multiple de type FDR (False Discovery Rate) a été appliquée.

I Résultats : Plusieurs métabolites étaient associés au risque de cancer du sein. La finalisation de l'identification des métabolites discriminants est en cours. Au vu des résultats actuels, les femmes caractérisées par un niveau plasmatique plus élevé de valine $(O R=1,45[1,15-1,83] ; p=0,009)$, de phénylalanine $(O R=1,43[1,14-1,78]$; $p=0,009)$, de tryptophane (OR=1,4 $[1,1-1,79] ; p=0,01)$, et de glutamine $(O R=1,33[1,07-1,66] ; p=0,01)$ et d'un niveau plus bas d'O-succinyl-L-homosérine $(O R=0,7[0,55-0,89] ; p=0,01)$ auraient un risque plus important de développer un cancer du sein dans la décennie qui suit.

I Conclusions et perspectives: Cette étude suggère plusieurs associations entre profils métabolomiques à l'inclusion et risque à long terme de développer le cancer du sein et confirme certains résultats que nous avons mis en évidence par une analyse RMN. Cette étude pourrait aider à améliorer l'identification des femmes à risque de développer un cancer du sein, bien avant l'apparition des symptômes. L'étude des biomarqueurs nutritionnels associés au risque de développer cette pathologie (actuellement en cours) pourra permettre de préciser le rôle de la nutrition (facteur modifiable) dans l'étiologie du cancer du sein et d'améliorer les stratégies préventives. 